\title{
The effects of a life skills-based intervention on emotional health, self-esteem and coping mechanisms in Malaysian institutionalised adolescents: protocol of a multi-centre randomized controlled trial
}

\begin{abstract}
The aim of the current study was to determine if a life skills-based intervention programme could improve the emotional health, self-esteem and coping skills of Malaysian institutionalised adolescents. This study was a randomized controlled trial with pre-, postand four-month follow-up tests comprising intervention and control groups which were randomly selected to receive the intervention programme (life skills education) or the placebo education programme (COMBI programme). Overall, 271 adolescents, who experienced a mild to extra severe score in one of the objectives of the DASS21 questionnaire were selected to participate in the study. The findings of this study can be the starting point of continuous, systematic life skills education for Malaysian children and adolescents, and can be used by researchers and educational planners across the country.
\end{abstract}

Keyword: Life skills education; Depression; Anxiety; Stress; Self-esteem; Coping mechanisms; Randomized controlled trial; Institutionalised adolescents; Malaysian adolescents 